\title{
The Ohdo blepharophimosis syndrome: a third case
}

\author{
Leslie G Biesecker
}

\begin{abstract}
A patient with a syndrome consisting of blepharophimosis, simple ears, hypoplastic teeth, developmental delay, and hypotonia is described. Previous case reports are reviewed and a differential diagnosis is described. Many of the features in the subject are similar to those described in two previous reports and they constitute a distinct syndrome.
\end{abstract}

Previous reports by Ohdo et al $^{1}$ and Say and Barber ${ }^{2}$ have documented the existence of a syndrome which includes dysmorphic facial features (most prominently blepharophimosis), mental retardation, and limb anomalies. An additional patient is described and a review of the differential diagnosis of blepharophimosis and mental retardation follows.

\section{Case report}

The patient was 4 years old when admitted with a diagnosis of periorbital cellulitis. He was the first born of a 30 year old gravida 2 para 2 woman and a 32 year old father. The pregnancy was complicated by polyhydramnios and anaemia of unknown aetiology. His birth weight was $3350 \mathrm{~g}$. At birth he was noted to have dysmorphic facial features, hypotonia, cryptorchidism, and limb anomalies. He required assisted ventilation for four days and was discharged from the nursery at 1 week of age. He was described as sucking poorly. Developmental delay was noted during the first year of life. At 26 months of age the patient had a febrile convulsion. A cranial CT scan and an EEG were normal. At 27 months of age he had an unsuccessful orchidopexy. A recent developmental assessment assigned an IQ of 54 to 56 .

He has a healthy, younger half sib and no other members of the family are known to be affected with a similar disorder. Both parents suffer from autoimmune disorders; his mother has hypothyroidism, erythema nodosum, and a positive antinuclear antibody (ANA)

Department of Pediatrics and Communicable Diseases, University of Michigan Medical Center, D1109 MPB Box 0718, Ann Arbor, MI 48109-0718, USA.

L G Biesecker

Received for publication 5 June 1990 .

Accepted for publication 4 July 1990 . test, and his father has ANA positive rheumatoid arthritis.

On physical examination his weight was $16.6 \mathrm{~kg}$ ( $\sim 50$ th centile), his head circumference was $51.7 \mathrm{~cm}$ (50th to 75th centile), and his height was estimated to be $103 \mathrm{~cm}$ ( 50th centile). He had marked blepharophimosis (palpebral fissures $2 \cdot 1 \mathrm{~cm}$ ) with telecanthus (inner canthal distance $4 \cdot 2 \mathrm{~cm}$ ) (figure). Bilateral optic atrophy was present and was more severe on the left. Other facial features included a broad and flat nasal bridge, a simple philtrum, and a thin vermilion. His ears were short $(4.5 \mathrm{~cm})$, the canals were stenotic, and the helices were simple. Micrognathia and a high arched palate were present. His teeth were conical in shape and small. He had a mild thoracic kyphoscoliosis. No heart murmur was noted. His scrotum was hypoplastic and the testes could not be palpated. The fingers appeared slender and the fifth was short and incurved. The metacarpophalangeal joints had decreased mobility and the wiists and elbows had increased mobility. The second and fourth toes overlapped the third and the ankles had decreased mobility. Flexion contractures of the knees and hips were also present. Two $5 \mathrm{~mm}$ café au lait spots and one hypopigmented patch were noted on the trunk.



Facial features of the proband include blepharophimosis, wide and flat nasal bridge, small and simple ears, and small teeth. 
Muscle tone was hypotonic and there was ankle clonus. A peripheral blood karyotype (approximately 675 band level resolution, performed in 1985) was reviewed and was normal.

\section{Discussion}

The patient reported here shares many features with the patients reported by Ohdo $e t a l^{1}$ and Say and Barber. ${ }^{2}$ The three patients reported by Ohdo et al ${ }^{1}$ were two sibs and their first cousin. They had blepharophimosis with ptosis and amblyopia, mental retardation (IQ range 37 to 42), hypoplastic teeth, hearing impairment, proteinuria, and congenital heart disease. In addition, the proband was noted to have auricular stenosis with a preauricular tag. The proband, her sister, and their father had normal karyotypes (550 band level resolution). Say and Barber ${ }^{2}$ reported a 3 year old boy with mental retardation (DQ $\sim 50$ ), blepharophimosis, blepharoptosis, hypoplastic teeth, myopia, nystagmus, midfacial hypoplasia, small, low set ears, laterally displaced, inverted nipples, hypoplastic scrotum with undescended testes, hyperextensible joints, fifth finger clinodactyly, hypoplastic thenar eminences, hypotonia, and a dislocated patella. No heart murmur was noted and the karyotype was not reported. The salient features of these five patients are listed in table 1.

The differential diagnosis of mental retardation with blepharophimosis includes the disorders listed in table 2. These disorders are compared with the physical features of the above patient as well as the four previously reported cases. For the purposes of discussion, the five patients will be designated as having the Ohdo blepharophimosis syndrome (OBS).

These five patients obviously do not suffer from one of the chromosomal syndromes listed in table 2 as these would have been detected in the 550 band level karyotype reported by Ohdo $e t a l^{1}$ and the higher
Table 2 The differential diagnosis of blepharophimosis and mental retardation.

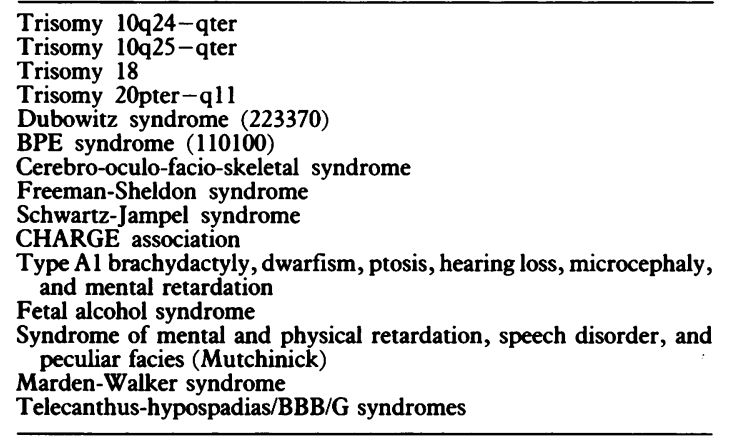

resolution study reported here. Microcephaly and hypospadias are major diagnostic features of Dubowitz syndrome ${ }^{3}$ and both of these features are absent in the patient reported here. None of the four previously reported OBS patients was microcephalic and the patient of Say and Barber ${ }^{2}$ was not noted to have hypospadias (the only male previously reported). The blepharophimosis, ptosis, epicanthus inversus syndrome (BPES) ${ }^{4}$ shows some similarity in facial features to the five OBS patients. BPES is not generally associated with mental retardation, deafness, abnormal muscle tone, contractures, or hypoplastic teeth, and the facial features in the OBS patients are more severe and mask-like than the BPES patients. Patients with the cerebro-oculo-facio-skeletal (COFS) syndrome $^{5}$ may have both blepharophimosis and central nervous system dysfunction, but this dysfunction is remarkable for arthrogryposis as well as progressive deterioration, which is absent in all five cases of OBS. In addition, the facial dysmorphology is distinct in that COFS syndrome patients have a prominent nasal root, marked micrognathia, and large

Table 1 Features of the five OBS patients.

\begin{tabular}{|c|c|c|c|c|c|}
\hline & Case $1^{1}$ & Case $2^{1}$ & Case $3^{1}$ & Case $4^{2}$ & $\begin{array}{c}\text { Case } 5 \\
\text { (This report) }\end{array}$ \\
\hline Blepharophimosis & + & + & + & + & + \\
\hline Wide/depressed nasal bridge & + & + & + & + & + \\
\hline Auricular stenosis & + & - & & & + \\
\hline Small pinnae & & & & + & + \\
\hline Deafness & + & + & + & + & + \\
\hline Microstomia & + & + & + & + & + \\
\hline Dental hypoplasia & + & + & + & + & + \\
\hline Developmental delay & + & + & + & + & + \\
\hline Heart defect & + & + & - & - & - \\
\hline Cryptorchidism & & & & + & + \\
\hline Proteinuria & + & + & + & + & \\
\hline Hyperextensible joints & & & & + & + \\
\hline Incurved fifth finger & & & & + & + \\
\hline Height & $-1.8 \mathrm{SD}$ & $-1.5 \mathrm{SD}$ & Normal & & Mean \\
\hline Weight & $-1 \cdot 1 \mathrm{SD}$ & $-0.5 \mathrm{SD}$ & Normal & & Mean \\
\hline Head circumference & $+1.9 \mathrm{SD}$ & Mean & Normal & & Mean \\
\hline
\end{tabular}

Mean=measurement near mean for age. 
ears with microcephaly. The facial features in Freeman-Sheldon syndrome are distinct from the OBS patients because of their mask-like, whistling facies with microstomia, long philtrum, hypoplastic nasal alae, and dimpled chin. ${ }^{6}$ The Schwartz-Jampel syndrome commonly includes blepharophimosis and, less frequently, mental retardation. ${ }^{7}$ However, progressive myotonia, joint limitation, and pectus carinatum are prominent features which distinguish these patients from the OBS patients. CHARGE is a non-random association of birth defects which includes the colobomatous malformation sequence, mental deficiency, heart defects, choanal atresia, growth deficiency, male genital hypoplasia, and ear anomalies (including deafness). ${ }^{8}$ The colobomatous malformation sequence may include blepharophimosis but this would be expected to be secondary to significant microphthalmia which was noted in only one of the OBS patients. Although the OBS patients have several CHARGE features (heart defect, mental deficiency, mild growth deficiency, and hearing deficiency), their distinct facial dysmorphology and hypoplastic teeth argue against inclusion in this diagnostic group. The syndrome with type Al brachydactyly, dwarfism, ptosis, hearing loss, microcephaly, and mental retardation reported by $T$ sukahara et $a \varphi^{9}$ is inconsistent with the long, thin fingers, normal stature, and normocephaly of the patient reported here. In addition, none of the four previously reported OBS patients have been reported to have brachydactyly, severe short stature, or microcephaly. The fetal alcohol syndrome ${ }^{10}$ should always be considered in any patient with developmental delay and ptosis or blepharophimosis. Again, the facial pattern is clearly distinct from the OBS patients and none of the case reports mentions significant alcohol consumption. The disorder reported by Mutchinick $e t$ $a l^{11}$ features severe microcephaly, a non-depressed nasal bridge, large ears, and no dental hypoplasia, which is clearly distinct from the OBS patients. The OBS patients have some similarities to the descriptions of patients with the Marden-Walker syndrome, ${ }^{12} 13$ although the pigeon breast, growth retardation, and microcephaly present in Marden-Walker syndrome are not present in the OBS patients. In addition, Marden-Walker syndrome is not associated with hypoplastic teeth and the nasal tip is prominent in contrast to the wide, flat nose with prominent lateral nasal tissue that is seen in the OBS patients. The $\mathrm{G}$ or telecanthus-hypospadias syndrome ${ }^{14}$ is not a likely diagnosis since the OBS patients lack hypospadias, cleft lip and palate, and urinary tract malformations, and the severe blepharophimosis of OBS is not a feature of the $G$ syndrome. This comparison with disorders which include blepharophimosis and mental retardation leads this author to conclude that the Ohdo blepharophimosis syndrome is indeed a distinct syndrome.
While the present case and that described by Say and Barber ${ }^{2}$ offer little insight into the inheritance pattern of this disorder, the report of Ohdo et $a l^{1}$ is puzzling in this respect. They propose that the pedigree is compatible with multifactorial, autosomal recessive, and autosomal dominant modes of inheritance. It is reasonable to propose multifactorial or autosomal dominant inheritance (with variable penetrance), but it is unlikely that a rare autosomal recessive disorder would affect cousins in the absence of consanguinity or non-paternity. Mitochondrial or $\mathrm{X}$ linked inheritance is unlikely with transmission through the father in the report of Ohdo et al. ${ }^{1}$ Although it is possible that a submicroscopic chromosomal abnormality could be present in the subject of this report and the case of Say and Barber, ${ }^{2}$ the cases of Ohdo et $a l^{1}$ would be incompatible with this aetiology. In conclusion the patient in this report is similar to those reported by Ohdo et $a l^{1}$ and Say and Barber $^{2}$ and they constitute a unique syndrome which may have an autosomal dominant or multifactorial aetiology.

\section{Note added in proof}

Buntinx and Majewski ${ }^{15}$ have recently reported a patient with blepharophimosis, iris coloboma, microgenia, hearing loss, postaxial polydactyly, aplasia of the corpus callosum, hydroureter, and developmental delay. This patient is distinct from the five patients with OBS but should be included in the differential diagnosis for blepharophimosis.

The author wishes to thank Drs Mason Barr and Robert Erickson for critical review of the manuscript and Dr Kamar for performing the dental evaluation.

1 Ohdo S, Madokoro H, Sonoda T, Hayakawa K. Mental retardation associated with congenital heart disease, blepharophimosis, blepharoptosis, and hypoplastic teeth. $\mathcal{F}$ Med Genet 1986;23:242-4.

2 Say B, Barber N. Mental retardation with blepharophimosis. F Med Genet 1987;24:511.

3 Winter RM. Dubowitz syndrome. F Med Genet 1986;23:11-3.

4 Oley C, Baraitser M. Blepharophimosis, ptosis, epicanthus inversus syndrome (BPES syndrome). F Med Genet 1988;25: 47-51.

5 Pena SDJ, Shokeir MHK. Autosomal recessive cerebro-oculofacio-skeletal syndrome. Clin Genet 1974;5:285-93.

6 Antley RM, Uga N, Burzynski NJ, Baum RS, Bixler D. Diagnostic criteria for the whistling face syndrome. Birth Defects 1975;11:161-8.

7 Edwards WC, Root AW. Chondrodystrophic myotonia (SchwartzJampel syndrome): report of a new case and follow-up of patients initially reported in 1969. Am $\mathcal{F}$ Med Genet 1982;13: $51-6$.

8 Hall BD. Choanal atresia and associated multiple anomalies. F Pediatr 1979;95:395-8.

9 Tsukahara M, Azuno Y, Kajii T. Type Al brachydactyly, dwarfism, ptosis, mixed partial hearing loss, microcephaly, and mental retardation. Am $\mathcal{F}$ Med Genet 1989;33:7-9.

10 Clarren SK, Smith DW. The fetal alcohol syndrome. N Engl f Med 1978;298:1063-7.

11 Mutchinick O. A syndrome of mental and physical retardation, speech disorders, and peculiar facies in two sisters. $\mathcal{f}$ Med Genet 1972;9:60-3. 
12 Howard FM, Rowlandson P. Two brothers with the MardenWalker syndrome: case report and review. $\mathcal{F}$ Med Genet 1981;18:50-3.

13 Gossage D, Perrin JM, Butler MG. A 26-month-old child with Marden-Walker syndrome and pyloric stenosis. Am 7 Med Genet 1987;26:915-9.
14 Cordero JF, Holmes LB. Phenotypic overlap of the BBB and G syndromes. Am F Med Genet 1978;2:145-52.

15 Buntinx I, Majewski F. Blepharophimosis, iris coloboma, microgenia, hearing loss, postaxial polydactyly, aplasia of corpus callosum, hydroureter, and developmental delay. Am $\mathcal{F}$ Med Genet 1990;36:273-4. 